\title{
Perfectively Deducing Bessel Mean Square Error Formula
}

\author{
Deng Yonghe ${ }^{* 1,2,3}$
}

${ }^{I}$ College of Engineering and designing, Lishui College, Lishui, Zhejiang, 323000, P.R. China

${ }^{2}$ School of Geodesy and Geomatics, Wuhan University, Wuhan 430079, P.R. China

${ }^{3}$ School of Continuous Education, Wuhan University, Wuhan 430079, P.R. China

\begin{abstract}
In the survey teaching materials of China, deducing Bessel mean square error formula is all based on survey values with same mathematical expectation. These methods aren't perfect. So, based on survey values without same mathematics expectation to prove Bessel mean square error formula is very necessary. Therefore, considering different mathematical expectation, it is meaningful that this paper has perfectively deduced Bessel mean square error formula.
\end{abstract}

Keywords: Bessel mean square error formula, survey values with different mathematics expectation, deducing.

\section{INTRODUCTION}

Bessel mean square error formula

$m= \pm \sqrt{\frac{[v v]}{n-1}}$

is a sort of basic formula about survey error. Where, $m$ is mean square error, $n$ is quantity of survey values, $v_{i}$ $(i=1,2, \cdots, n)$ is residual of survey values, and in survey teaching materials, $[v v]$ generally represents $\sum_{i=1}^{n} v_{i}^{2}$. (Behind of this paper, the meaning of $[v v]$ still is $\sum_{i=1}^{n} v_{i}^{2}$.).

But, when we deduce Bessel mean square error formula in survey teaching materials of China, we often select survey values with same mathematics expectation [1-18], and generally survey values with different mathematics expectation are not selected. The literatures $[4,6,8,12-15,17]$ are all national programming teaching materials of Chinese common higher education, and literatures $[12,13,15,17]$ are all called as Chinese excellent surveying teaching materials. But these methods may give students viewpoint that Bessel mean square error formula based on survey values with different mathematics expectation cannot be deduced. Though the viewpoint is wrong, because nobody has finished generally deducing Bessel mean square error formula, but the viewpoint is still used for Bessel mean square error formula, and influenced survey teaching materials and students of China. So, in this paper, it is meaningful to perfectly deduce Bessel mean square error formula.

*Address correspondence to this author at College of Engineering and Designing, Lishui College, Lishui, Zhejiang, 323000, P.R. China;

Tel: +86 18969588403;E-mail: 1sxydengyonghe@sina.com, a15925722009@163.com

\section{PERFECTIVELY DEDUCING BESSEL MEAN SQUARE ERROR FORMULA}

If there are equal precision independent surveying values such as $l_{1}, l_{2}, \cdots, l_{n}$, their residuals are $v_{1}, v_{2}, \cdots, v_{n}$, their mathematics expectation are $\mu_{1}, \mu_{2}, \cdots, \mu_{n}$, their variance is $\sigma^{2}$, and their density is [19]

$f\left(l_{i}\right)=\frac{1}{\sqrt{2 \pi} \sigma} \exp \frac{-\left(l_{i}-\mu_{i}\right)^{2}}{2 \sigma^{2}} \quad(i=1,2, \cdots, n)$

2.1. $\mu_{1} \neq \cdots \neq \mu_{i} \neq \cdots \neq \mu_{n}(i=1,2, \cdots, n)$ isn't true

When $\mu_{1} \neq \cdots \neq \mu_{i} \neq \cdots \neq \mu_{n}(i=1,2, \cdots, n)$ isn't true, based on Eq.2, we can receive maximum likelihood function[19].

$f\left(l / \mu_{1}, \mu_{2}, \cdots, \mu_{n}, \sigma^{2}\right)=\prod_{i=1}^{n} f\left(l_{i}\right)=\frac{1}{(\sqrt{2 \pi})^{n} \sigma^{n}} \exp \left[-\sum_{i=1}^{n} \frac{\left(l_{i}-\mu_{i}\right)^{2}}{2 \sigma^{2}}\right]$

Then,

$\ln f\left(l / \mu_{1}, \mu_{2}, \cdots, \mu_{n}, \sigma^{2}\right)=-n \ln \sqrt{2 \pi}-\frac{n}{2} \ln \sigma^{2}-\sum_{i=1}^{n} \frac{\left(l_{i}-\mu_{i}\right)^{2}}{2 \sigma^{2}}$

2.1.1. $\mu_{i}(i=1,2, \cdots, n)$ is already known

From Eq.4, we can get

$\left.\frac{\partial \ln f\left(l / \mu_{1}, \mu_{2}, \cdots, \mu_{n}, \sigma^{2}\right)}{\partial \sigma^{2}}\right|_{\sigma^{2}=\hat{\sigma}^{2}}=-\frac{n}{2 \hat{\sigma}^{2}}+\sum_{i=1}^{n} \frac{\left(l_{i}-\mu_{i}\right)^{2}}{2 \hat{\sigma}^{4}}=0$

Therefore; 


$$
\hat{\sigma}^{2}=\frac{\sum_{i=1}^{n}\left(l_{i}-\mu_{i}\right)^{2}}{n}
$$

Then;

$m=\hat{\sigma}= \pm \sqrt{\frac{\sum_{i=1}^{n}\left(l_{i}-\mu_{i}\right)^{2}}{n}}= \pm \sqrt{\frac{\sum_{i=1}^{n} v_{i}^{2}}{n}}= \pm \sqrt{\frac{[v v]}{n}}$

If we want Eq.7 to have an unprejudiced character, we should change Eq.7 into Eq.8

$$
m= \pm \sqrt{\frac{[v v]}{n-1}}
$$

\subsection{2. $\mu_{i}(i=1,2, \cdots, n)$ isn't already known}

From Eq.4, we can get

$$
\left.\frac{\partial \ln f\left(l / \mu_{1}, \mu_{2}, \cdots, \mu_{n}, \sigma^{2}\right)}{\partial \sigma^{2}}\right|_{\sigma^{2}=\hat{\sigma}^{2}, \mu_{i}=\hat{\mu}_{i}}=-\frac{n}{2 \hat{\sigma}^{2}}+\sum_{i=1}^{n} \frac{\left(l_{i}-\hat{\mu}_{i}\right)^{2}}{2 \hat{\sigma}^{4}}=0
$$

$$
\left.\frac{\partial \ln f\left(l / \mu_{1}, \mu_{2}, \cdots, \mu_{n}, \sigma^{2}\right)}{\partial \mu_{i}}\right|_{\sigma^{2}=\hat{\sigma}^{2}, \mu_{i}=\hat{\mu}_{i}}=\frac{l_{i}-\hat{\mu}_{i}}{\hat{\sigma}^{2}}=0
$$

Therefore;

$$
\begin{gathered}
\hat{\sigma}^{2}=\frac{\sum_{i=1}^{n}\left(l_{i}-\hat{\mu}_{i}\right)^{2}}{n} \\
\hat{\mu}_{i}=l_{i}(i=1,2, \cdots, n)
\end{gathered}
$$

From Eq.11, we can get

$$
m=\hat{\sigma}= \pm \sqrt{\frac{\sum_{i=1}^{n}\left(l_{i}-\hat{\mu}_{i}\right)^{2}}{n}}= \pm \sqrt{\frac{\sum_{i=1}^{n} v_{i}^{2}}{n}}= \pm \sqrt{\frac{[v v]}{n}}
$$

If we want Eq.13 to have an unprejudiced character, we should change Eq.13 into Eq.14

$m= \pm \sqrt{\frac{[v v]}{n-1}}$

So, we can say that when $\mu_{1} \neq \cdots=\mu_{i} \neq \cdots \neq \mu_{n}$ ( $i=1,2, \cdots, n)$ isn't true, and they aren't already known, Bessel mean square error formula has been testified in this paper. But, no matter how bad or good survey values are, when we take Eq.12 into Eq.14 or Eq.13, we can only obtain in a certain case:

$m=0$
This is a kind of special circumstance.

2.2. $\mu_{1}=\cdots=\mu_{i}=\cdots=\mu_{n}(i=1,2, \cdots, n)$ is true

Because independent surveying values $\left(l_{1}, l_{2}, \cdots, l_{n}\right)$ have equal precision, and $\mu_{1}=\cdots=\mu_{i}=\cdots=\mu_{n}($ $i=1,2, \cdots, n)$ is true, we can assume survey values come from same mother body and assume $\mu_{i}=\mu(i=1,2, \cdots, n)$. So, we can receive maximum likelihood function based on Eq.2 [19].

$f\left(l / \mu_{1}, \mu_{2}, \cdots, \mu_{n}, \sigma^{2}\right)=\prod_{i=1}^{n} f\left(l_{i}\right)=\frac{1}{(\sqrt{2 \pi})^{n} \sigma^{n}} \exp \left[-\sum_{i=1}^{n} \frac{\left(l_{i}-\mu\right)^{2}}{2 \sigma^{2}}\right]$

Then;

$\ln f\left(l / \mu_{1}, \mu_{2}, \cdots, \mu_{n}, \sigma^{2}\right)=-n \ln \sqrt{2 \pi}-\frac{n}{2} \ln \sigma^{2}-\sum_{i=1}^{n} \frac{\left(l_{i}-\mu\right)^{2}}{2 \sigma^{2}}$

\subsection{1. $\mu_{i}(i=1,2, \cdots, n)$ is already known}

When $\mu_{i}(i=1,2, \cdots, n)$ is already known, we can get Eq. 18 from Eq. 17

$$
\left.\frac{\partial \ln f\left(l / \mu_{1}, \mu_{2}, \cdots, \mu_{n}, \sigma^{2}\right)}{\partial \sigma^{2}}\right|_{\sigma^{2}=\hat{\sigma}^{2}}=-\frac{n}{2 \hat{\sigma}^{2}}+\sum_{i=1}^{n} \frac{\left(l_{i}-\mu\right)^{2}}{2 \hat{\sigma}^{4}}=0
$$

Therefore;

$$
\hat{\sigma}^{2}=\frac{\sum_{i=1}^{n}\left(l_{i}-\mu\right)^{2}}{n}
$$

Then;

$m=\hat{\sigma}= \pm \sqrt{\frac{\sum_{i=1}^{n}\left(l_{i}-\mu\right)^{2}}{n}}= \pm \sqrt{\frac{\sum_{i=1}^{n} v_{i}^{2}}{n}}= \pm \sqrt{\frac{[v v]}{n}}$

If we want Eq.20 to have an unprejudiced character, we should change Eq.20 into Eq.21

$m= \pm \sqrt{\frac{[v v]}{n-1}}$

\subsection{2. $\mu_{i}(i=1,2, \cdots, n)$ isn't already known}

When $\mu_{1}=\cdots=\mu_{i}=\cdots=\mu_{n}(i=1,2, \cdots, n)$ is true, and they aren't already known, the paper [20] has deduced Bessel mean square error formula.

\section{CONCLUSIONS}

In survey teaching materials of China, deducing Bessel mean square error formula is based on survey values with same mathematics expectations. Those teaching materials haven't solved survey values with different mathematics expectation so it isn't deemed to be perfect. 
Based on survey values which don't have the same mathematics expectation, this paper has put forward a sort of method to prove Bessel mean square error formula. The method has solved problems which haven't been solved by survey teaching materials of China, include national programming teaching materials of Chinese common higher education and Chinese excellent surveying teaching materials.

In addition, the paper has studied all sorts of circumstances to deduce Bessel mean square error formula. So, the paper is complete.

Obviously,the paper is good for Bessel mean square error formula to be perfective, for students to understand Bessel mean square error formula, for survey teaching materials of China, include national programming teaching materials of Chinese common higher education and Chinese excellent surveying teaching materials, to be renewed

\section{ABOUT THE AUTHORS}

Author: Deng Yonghe, College of Engineering and Designing of Lishui College. The author's major is data processing and higher education.58 papers had been published, and among them, 55 papers had independently been finished.

\section{CONFLICT OF INTEREST}

The author confirms that this article content has no conflict of interest.

\section{ACKNOWLEDGEMENTS}

Declared none.

\section{REFERENCES}

[1] Z.K. Feng,L. Zhou, J.L. Zhao and C.-J. Zhao, Principle of Surveying, Chinese Forestry Press: Beijing, 2002, pp. 44-45.
[2] X.Q. Fu, Surveying, Beijing University of Technology Press: Beijing, 2006, pp. 78-80

[3] N.W. Deng, Surveying, $2^{\text {nd }}$ ed., China Electric Power Press: Beijing, 2010, pp. 95-96

[4] X.W. Cheng, X.Y. Chen, G. Chen, Z.T. Ming and J.T. Xu, Surveying, Geological Publishing House: Beijing, 2008, pp. 62-64.

[5] Surveying edition group of Hehai University, Surveying, $2^{\text {nd }}$ ed. National Defence Industry Press: Beijing, 2013, pp. 83-84.

[6] T.W. Li, et al., Model Surveying, $2^{\text {nd }}$ ed., Science Press: Beijing, 2014, pp. 132-134.

[7] Z.Q. Luo, Surveying, $2^{\text {nd }}$ ed., Yunnan University Press: Kunming, 2010, pp. 94-97.

[8] L. Chen and H.F. Lou, Model Surveying Technique, China Water \& Power Press: Beijing, 2011, pp. 92-94

[9] S.L. Yang, T.F. Yang and H.Y. Shi, Surveying, $2^{\text {nd }}$ ed., Press of China Railway: Beijing, 2013, pp. 118-120.

[10] X.M. Zhang, X.L. Li and Z.X. Liao, Surveying, Press of Heifei University of Technology: Heifei, 2007, pp. 96-98.

[11] L.H. Chen, G.B. Du, H. Zhang and L.R. Zhao, Surveying, Zhe jiang University Press: Hangzhou, 2009, pp. 96-99.

[12] N. Wang and J.J. Guo, Modern General Surveying, $2^{\text {nd }}$ ed., Tsinghua University Press: Beijing, 2009, pp. 114-116.

[13] X.L. Gu, F. Bao and X.J. Cheng, Surveying, $4^{\text {th }}$ ed., Tongji University Press: Tongji, 2011, pp. 126-127.

[14] Z.F. Pan, Z.Y. Yang, X.J. Cheng, S. Cheng and T.J. Wang, Principles and Method of Digital Mapping, Wuhan University Press: Wuhan, 2004, pp. 48-50.

[15] Z.F. Pan, X.J. Cheng, S. Cheng, T.J. Wang, W.D. Song and J.G. Zou, Principles and Method of Digital Mapping, $2^{\text {nd }}$ ed., Wuhan University Press: Wuhan, 2011, pp. 30-32.

[16] H. Qin, X. Wu, P.Y. Tang and D.J. Yu, Civil Engineering Surveying, $4^{\text {th }}$ ed., Tongji University Press: Tongji, 2013, pp. 141143.

[17] J.X. Gao, B.L. Xiao, P.Y. Fu, X.X. Yu, M. Liang, H.Y. Liang, F.L. Yang and G.L. Chen, Principles and Method of Digital Mapping, China University Of Mining And Technology: Xuzhou, 2010, pp. 100-102

[18] T.S. Wang and T.Q. Yuan, Basic Surveying, Press of Yellow River Water Conservancy: Zhengzhou, 2008, pp. 115-117.

[19] X.Z. Cui, Z.C. Yu, B.Z. Tao, D.J. Liu, Z.L. Yu, H.Y. Sun and X.Z Wang, Generalized Surveying Adjustment, new ed., Wuhan Technical University of Surveying and Mapping Press: Wuhan, 2001, pp. 2-10

[20] Y. H. Deng, "Deduction of Bessel mean square error formula," Journal of Geodesy and Geodynamics, vol. 29, pp. 128-130, 2009.

Received: October 8,2014

Revised: October 25, 2014

Accepted: December 23, 2014

(C) Deng Yonghe; Licensee Bentham Open

This is an open access article licensed under the terms of the Creative Commons Attribution Non-Commercial License (http://creativecommons.org/licenses/ by-nc/3.0//) which permits unrestricted, non-commercial use, distribution and reproduction in any medium, provided the work is properly cited. 